\title{
FINITE VOLUME BOX SCHEMES AND MIXED METHODS
}

\author{
JEAN-PierRe Croisille ${ }^{1}$
}

\begin{abstract}
We present the numerical analysis on the Poisson problem of two mixed Petrov-Galerkin finite volume schemes for equations in divergence form $\operatorname{div} \varphi(u, \nabla u)=f$. The first scheme, which has been introduced in [22], is a generalization in two dimensions of Keller's box-scheme. The second scheme is the dual of the first one, and is a cell-centered scheme for $u$ and the flux $\varphi$. For the first scheme, the two trial finite element spaces are the nonconforming space of Crouzeix-Raviart for the primal unknown $u$ and the div-conforming space of Raviart-Thomas for the flux $\varphi$. The two test spaces are the functions constant per cell both for the conservative and for the flux equations. We prove an optimal second order error estimate for the box scheme and we emphasize the link between this scheme and the post-processing of Arnold and Brezzi of the classical mixed method.
\end{abstract}

Résumé. Nous effectuons l'analyse numérique pour le problème de Poisson de deux schémas volumes finis mixtes de type Petrov-Galerkin pour des équations sous forme divergence $\operatorname{div} \varphi(u, \nabla u)=f$. Le premier schéma, qui a été introduit dans [22], est une généralisation à deux dimensions du schéma boîte de Keller. Le second schéma, dual du premier, est de type "cell-center" pour $u$ et pour le flux $\varphi$. Dans le premier schéma, les deux espaces d'approximation sont l'espace non conforme de Crouzeix-Raviart pour l'inconnue primale $u$ et l'espace de Raviart-Thomas pour le flux $\varphi$. Les deux espaces test sont les espaces des fonctions constantes par cellule, à la fois pour l'équation conservative et l'équation du flux. Nous prouvons une estimation d'erreur optimale en $O\left(h^{2}\right)$ pour le schéma boitte et nous mettons en évidence le lien entre ce schéma et le post-processing d'Arnold et Brezzi de la méthode mixte classique.

Mathematics Subject Classification. 35J25, 65P05, 73V05, 65M15, 65N30.

Received: November 12, 1999. Revised: May 10, 2000.

\section{INTRODUCTION}

In this paper, we perform the numerical analysis of two finite volume schemes for conservative equations. The first scheme is a "finite volume box scheme" introduced in [22]. This kind of scheme originates in the pioneering paper by H.B. Keller [31], where the basic principles of box-schemes are introduced on the model problem of the 1D heat equation. In the lowest order version, these principles are, for a problem in divergence form like $\operatorname{div} \varphi(u, \nabla u)=f$, firstly to use degrees of freedom located on the faces of the mesh (the edges in two dimensions) both for the unknown $u$ and the flux $\varphi$, secondly to build the discrete equations by averaging the continuous ones onto "boxes". In this sense, they are finite-volume schemes, that is, schemes ensuring a local conservation property at the level of the mesh.

Beside a direct use of Keller's scheme for variants of 1D heat equations in some works, [27,34], two types of "box-schemes" are known in the literature.

Keywords and phrases. Box method, box scheme, mixed finite element method, Petrov-Galerkin method, finite volume method.

1 Département de Mathématiques, Université de Metz, 57045 Metz, France. e-mail: croisil@poncelet.univ-metz.fr 
The first one has been introduced in computational fluid dynamics, especially the compressible Euler equations. The main idea is that locating the conservative unknowns (density, momentum, energy) at the center of the faces of the mesh is very natural in order to build a scheme discretizing both the conservative and the flux equations at the level of a single cell. We refer to [14-17, 19-21,41,42] where numerical results on Euler or Navier-Stokes equations are presented.

Another kind of box scheme is known under the name of "box method" or "finite volume element method". The design of this scheme is similar to the one of the so called "cell-vertex finite volume method", (see e.g. [28]). The unknown $u$ belongs to a finite element space such that $P^{1}$ or $Q^{1}$. The discrete equations are defined from averaging the continuous ones onto a dual box surrounding each vertex. We refer to $[4,13,30,32,37-39]$ and the references therein.

The scheme introduced in [22] for the 2D Poisson problem on a triangular mesh is a Keller-like scheme. Contrary to $[15,16,19]$ where a finite differencing interpretation of the discrete unknowns is used, a finite element interpretation of the degrees of freedom is introduced. On a regular FEM triangulation $\mathcal{T}_{h}$ by triangles $K$, the mixed form of the Poisson problem

$$
\begin{cases}\operatorname{div} p+f=0 & \text { in } \Omega \\ p-\nabla u=0 & \text { in } \Omega \\ u=0 & \text { on } \partial \Omega\end{cases}
$$

is approximated by the finite volume scheme (called $F$ Vbox in the sequel): find $\left(u_{h}, p_{h}\right) \in P_{n c, 0}^{1} \times R T^{0}$ such that

$$
\begin{cases}\left\langle\operatorname{div} p_{h}+f, \mathbb{1}_{K}\right\rangle=0 & \forall K \in \mathcal{T}_{h} \\ \left\langle p_{h}-\nabla u_{h}, \mathbb{1}_{K}\right\rangle=0 & \forall K \in \mathcal{T}_{h} \\ u_{h}=0 & \text { on } \partial \Omega .\end{cases}
$$

In (2), $P_{n c, 0}^{1}$ is the nonconforming space of Crouzeix-Raviart, [24], with homogeneous boundary conditions, and $R T^{0}$ is the div-conforming space of Raviart-Thomas of lowest order. Note that the coupling between these two spaces is unusual in the classical variational mixed methods theory, $[8,9,11,29]$, because this couple of spaces does not satisfy the Babuška-Brezzi condition.

The aim of this paper is to prove that the numerical analysis of (2) can be simply performed by using the classical theory of the mixed Petrov-Galerkin approximations, [6,7,35]. For simplicity of the notation, we restrict ourselves here to the academic Poisson problem in two dimensions, but generalizations to the 3D case or to more complex conservative problems are possible.

The outline of the paper is as follows. In Section 2 we introduce the mixed formulation of which the "FVbox" scheme is an approximation. In fact, this formulation generates also a second scheme, (called "dual FVbox"), which is a cell-centered finite volume scheme for the couple of unknowns $(u, \nabla u)$, and which is also apparently new. We verify that the theory of nonconforming mixed approximations applies to the two schemes. A second order estimate is derived in Section 2.4 for the FVbox scheme, using an Aubin-Nitsche argument. Finally, we prove in Section 3 that the FVbox scheme coincides in fact with one of the post-processings of Arnold and Brezzi of the classical mixed method [2], or equivalently, with the a posteriori interpretation of Marini [33].

Let us mention finally that several recent papers deal with the numerical analysis or the design of cellcentered finite volume methods with the help of the mixed finite element theory. A first kind of works, [5,25,43] is devoted to the a posteriori interpretation of the standard mixed solution $u_{h}$ as a cell-centered finite volume method. In [40], a new mixed finite volume method, different from the present one, is introduced and analyzed by the mixed Petrov-Galerkin theory. Finally, for a numerical analysis of the cell-centered finite volume method without reference to the FEM analogy, we refer to the exhaustive study [26]. See also [18].

This work was announced in [23]. 


\section{Numerical ANALYSis of the FVbox SCHEME}

\subsection{Two mixed forms of the Poisson problem}

We consider the bidimensional Poisson problem in mixed form in a bounded domain $\Omega \subset \mathbb{R}^{2}$

$$
(M) \quad \begin{cases}\operatorname{div} p+f=0 & \text { in } \Omega \\ p-\nabla u=0 & \text { in } \Omega \\ u=0 & \text { onto } \partial \Omega\end{cases}
$$

Let us recall some standard notation. We denote by $L^{2}$ the Hilbert space of square integrable functions on $\Omega$, equipped with the norm $|u|_{0, \Omega} . H^{1}, H^{2}$ are the standard Sobolev spaces equipped with their norms $\|u\|_{1}=\left(|u|_{0, \Omega}^{2}+|\nabla u|_{0, \Omega}^{2}\right)^{1 / 2}$ and $\|u\|_{2}=\left(\|u\|_{1, \Omega}^{2}+\left|D^{2} u\right|_{0, \Omega}^{2}\right)^{1 / 2} . H_{0}^{1}$ is the Hilbert space of functions $u \in H^{1}$ having a null trace onto $\partial \Omega$. The semi-norm $|u|_{1, \Omega}=|\nabla u|_{0, \Omega}$ is a norm on $H_{0}^{1}$ equivalent to $\|u\|_{1}$. Finally $H_{\text {div }}$ is the Hilbert space of bidimensional vector fields $p \in\left(L^{2}\right)^{2}$ such that $\operatorname{div} p \in L^{2}$, equipped with the norm $\|p\|_{\operatorname{div}, \Omega}=\left(|p|_{0, \Omega}^{2}+|\operatorname{div} p|_{0, \Omega}^{2}\right)^{1 / 2}$.

If $f \in L^{2}$ and $\Omega$ is $C^{2}$ or convex, this problem has a unique solution $u \in H_{0}^{1} \cap H^{2}$ which verifies $\|u\|_{2, \Omega} \leq C|f|_{0, \Omega}$.

We do not address here the well-known mixed formulations with unknowns $(u, p) \in H_{0}^{1} \times\left(L^{2}\right)^{2}$ or $(u, p) \in L^{2} \times H_{\text {div }}$, which are the continuous framework respectively of the classical conforming (or nonconforming) method and of the mixed method of Raviart and Thomas.

The two mixed formulations that are needed in the sequel are of Petrov-Galerkin type, that is, they use two different Hilbert spaces as primal and dual spaces. The first one is: find $(u, p) \in H_{0}^{1} \times H_{\text {div }}$ such that

$$
\left(P_{1}\right) \quad \begin{cases}(\operatorname{div} p+f, v)_{0, \Omega}=0 & \forall v \in L^{2} \\ (p-\nabla u, q)_{0, \Omega}=0 & \forall q \in\left(L^{2}\right)^{2}\end{cases}
$$

Note that this formulation is also introduced in [40]. The second-one is the dual of $\left(P_{1}\right)$. It reads: find $(v, q) \in L^{2} \times\left(L^{2}\right)^{2}$ such that

$$
\left(P_{2}\right) \quad \begin{cases}-(\nabla u, q)_{0, \Omega}+(f, u)_{0, \Omega}=0 & \forall u \in H_{0}^{1} \\ (p, q)_{0, \Omega}+(\operatorname{div} p, v)_{0, \Omega}=0 & \forall p \in H_{\mathrm{div}}\end{cases}
$$

These two formulations are connected with the bilinear continuous form $B[(u, p) ;(v, q)]$ defined for $(u, p) \in H_{1}=H_{0}^{1} \times H_{\text {div }},(v, q) \in H_{2}=L^{2} \times\left(L^{2}\right)^{2}$ by

$$
B[(u, p) ;(v, q)]=a(p, q)+b_{1}(q, u)+b_{2}(p, v)
$$

where $a$ is the continuous bilinear form defined on $H_{\mathrm{div}} \times\left(L^{2}\right)^{2}$ by

$$
a(p, q)=(p, q)_{0, \Omega}
$$

and $b_{1}, b_{2}$ are the two forms respectively defined for $(u, q) \in H_{0}^{1} \times\left(L^{2}\right)^{2}$ and $(v, p) \in L^{2} \times H_{\text {div }}$ by

$$
b_{1}(q, u)=-(\nabla u, q)_{0, \Omega}, \quad b_{2}(p, v)=(\operatorname{div} p, v)_{0, \Omega}
$$

We recall now briefly the following abstract result due to Babuška [3], Brezzi [10], Nicolaides [35], Bernardi et al. $[6,7]$, devoted to the abstract formulation of mixed problems. Suppose given four Hilbert spaces

$$
\left(X_{1},(\cdot, \cdot)_{X_{1}}\right),\left(X_{2},(\cdot, \cdot)_{X_{2}}\right),\left(M_{1},(\cdot, \cdot)_{M_{1}}\right),\left(M_{2},(\cdot, \cdot)_{M_{2}}\right)
$$


and

(a) a continuous bilinear form $a(\cdot, \cdot): X_{1} \times X_{2} \rightarrow \mathbb{R}$;

(b) two continuous bilinear forms $b_{1}(\cdot, \cdot): X_{2} \times M_{1} \rightarrow \mathbb{R}, b_{2}(\cdot, \cdot): X_{1} \times M_{2} \rightarrow \mathbb{R}$. We call $H_{1}, H_{2}$, the Hilbert spaces $H_{1}=X_{1} \times M_{1}, H_{2}=X_{2} \times M_{2}$ equipped with the norms

$$
\begin{aligned}
\|(u, \lambda)\|_{H_{1}} & =\left(\|u\|_{X_{1}}^{2}+\|\lambda\|_{M_{1}}^{2}\right)^{1 / 2} \\
\|(v, \mu)\|_{H_{2}} & =\left(\|v\|_{X_{2}}^{2}+\|\mu\|_{M_{2}}^{2}\right)^{1 / 2} .
\end{aligned}
$$

The null spaces $V_{1} \subset X_{1}, V_{2} \subset X_{2}$ are defined by

$$
\begin{array}{ll}
V_{1}=\left\{u \in X_{1} \mid b_{2}(u, \mu)=0\right. & \left.\forall \mu \in M_{2}\right\} \\
V_{2}=\left\{v \in X_{2} \mid b_{1}(v, \lambda)=0\right. & \left.\forall \lambda \in M_{1}\right\} .
\end{array}
$$

For any $l_{2} \in X_{2}^{\prime}, m_{2} \in M_{2}^{\prime}$, we consider the abstract Problem $(M)$ : find $(u, \lambda) \in X_{1} \times M_{1}$ such that

$$
(M) \begin{cases}a(u, v)+b_{1}(v, \lambda)=\left\langle l_{2}, v\right\rangle_{X_{2}^{\prime}, X_{2}} & \forall v \in X_{2} \\ b_{2}(u, \mu)=\left\langle m_{2}, \mu\right\rangle_{M_{2}^{\prime}, M_{2}} & \forall \mu \in M_{2} .\end{cases}
$$

Theorem 2.1. The Problem $(M)$ has a unique solution $(u, \lambda)$, with continuous dependence on the data $\left(l_{2}, m_{2}\right) \in X_{2}^{\prime} \times M_{2}^{\prime}$, if and only if the four following conditions hold:

(i) The bilinear form $a$ is such that for any $v \in V_{2}$

$$
\sup _{u \in V_{1},\|u\|_{X_{1}} \leq 1} a(u, v) \geq \alpha\|v\|_{X_{2}}
$$

where $\alpha>0$.

(ii) For any $u \in V_{1}$

$$
\forall v \in V_{2} \quad a(u, v)=0 \Rightarrow u=0
$$

(iii $\left.i_{1}\right)$ There exists $\beta_{1}>0$ such that for any $\lambda \in M_{1}$

$$
\sup _{v \in X_{2},\|v\|_{X_{2}} \leq 1} b_{1}(v, \lambda) \geq \beta_{1}\|\lambda\|_{M_{1}}
$$

(iii $i_{2}$ There exists $\beta_{2}>0$ such that for any $\mu \in M_{2}$

$$
\sup _{u \in X_{1},\|u\|_{X_{1}} \leq 1} b_{2}(u, \mu) \geq \beta_{2}\|\mu\|_{M_{2}}
$$

In addition, the conditions (i), (ii) are equivalent to the dual conditions ( ${ }^{\prime}$ ), (ii').

$\left(i\right.$ ) The bilinear form $a$ is such that for any $u \in V_{1}$,

$$
\sup _{v \in V_{2},\|v\|_{X_{2}} \leq 1} a(u, v) \geq \alpha^{\prime}\|u\|_{X_{1}}
$$

where $\alpha^{\prime}>0$.

(ii') For any $v \in V_{2}$,

$$
\forall u \in V_{1} \quad a(u, v)=0 \Rightarrow v=0 .
$$


Another couple of equivalent conditions is $(i)+\left(i^{\prime}\right)$. Therefore, the Problem $(M)$ is well posed if and only if, for any $\left(l_{1}, m_{1}\right) \in X_{1}^{\prime} \times M_{1}^{\prime}$, so is the dual problem $\left(M^{\prime}\right)$ : find $(v, \mu) \in X_{2} \times M_{2}$ such that

$$
\left(M^{\prime}\right) \quad \begin{cases}a(u, v)+b_{2}(u, \mu)=\left\langle l_{1}, u\right\rangle_{X_{1}^{\prime}, X_{1}} & \forall u \in X_{1} \\ b_{1}(v, \lambda)=\left\langle m_{1}, \lambda\right\rangle_{M_{1}^{\prime}, M_{1}} & \forall \lambda \in M_{1} .\end{cases}
$$

The well known particular case of Theorem 2.1 is when $X_{1}=X_{2}=X, M_{1}=M_{2}=M, b_{1}=b_{2}$. In this case, the two null spaces $V_{1}, V_{2}$ are identical and coincide with the space

$$
V=\{u \in X / b(u, \lambda)=0, \forall \lambda \in M\}
$$

Conditions (i), (ii), ( $\left.i i_{1,2}\right)$ for well-posedness of problem $(M)$ reduce to

(i) $\forall u \in V, \sup _{v \in V,\|v\|_{V} \leq 1} a(u, v) \geq \alpha \quad\|u\|_{X}$

(ii) for any $v \in V, \quad a(u, v)=0 \quad \forall u \in V \Rightarrow v=0$

(iii) $\forall \lambda \in M, \sup _{u \in X,\|u\|_{X} \leq 1} b(u, \lambda) \geq \beta\|\lambda\|_{M} \quad$ (LBB condition).

If the bilinear form $a$ is symmetric, the conditions $(i)+(i i)$ are clearly equivalent to the condition (i) alone. Furthermore, a sufficient condition in order to have $(i)+(i i)$ is the coercivity of the form $a$ restricted to the space $V \times V$, i.e.

$$
a(u, u) \geq \alpha\|u\|_{X}^{2} \quad \forall u \in V .
$$

We apply Theorem 2.1 to the two Problems $\left(P_{1}\right),\left(P_{2}\right)$ with the bilinear forms $a, b_{1}, b_{2}$ defined by $(7),(8)$. The Hilbert spaces are

$$
X_{1}=H_{\mathrm{div}}, \quad X_{2}=\left(L^{2}\right)^{2}, \quad M_{1}=H_{0}^{1}, \quad M_{2}=L^{2}
$$

equipped with their natural norms.

Proposition 2.2. The bilinear forms $a, b_{1}, b_{2}$ fulfill the conditions (i), (ii), (iii $\left.i_{1}\right)$, (iii $i_{2}$ ) of Theorem 2.1 .

Proof. The two properties $\left(i i_{1}\right),\left(i i_{2}\right)$ are precisely the (LBB) conditions for the couples of spaces $(X, M)=\left(\left(L^{2}\right)^{2}, H_{0}^{1}\right)$ and $(X, M)=\left(H_{\text {div }}, L^{2}\right)$ which are true, [8]. Furthermore, the two null spaces $V_{1}$, $V_{2}$ defined by

$$
\begin{array}{ll}
V_{1}=\left\{p \in H_{\operatorname{div}} /(\operatorname{div} p, u)_{0, \Omega}=0\right. & \left.\forall u \in L^{2}\right\} \\
V_{2}=\left\{p \in\left(L^{2}\right)^{2} /(p, \nabla u)_{0, \Omega}=0\right. & \left.\forall u \in H_{0}^{1}\right\}
\end{array}
$$

are identical and reduce to the space $V$ of square integrable vector fields, with null divergence in the space of distributions $\mathcal{D}^{\prime}(\Omega)^{2}$. For $p \in V, a(p, p)=|p|_{0, \Omega}^{2}=\|p\|_{\operatorname{div}, \Omega}^{2}$. Therefore, the restriction of the form $a$ to $V \times V$ is coercive, which gives the result.

We deduce from Theorem 2.1 the following result.

Corollary 2.3. For any $f \in L^{2}$

(i) there exists a unique solution $(u, p) \in H_{0}^{1} \times H_{\mathrm{div}}$ of $\left(P_{1}\right)$ such that

$$
\|u\|_{1, \Omega}+\|p\|_{\operatorname{div}, \Omega} \leq C_{1}|f|_{0, \Omega}
$$


(ii) there exists a unique solution $(v, q) \in L^{2} \times\left(L^{2}\right)^{2}$ solution of $\left(P_{2}\right)$ such that

$$
|v|_{0, \Omega}+|q|_{0, \Omega} \leq C_{2}|f|_{0, \Omega}
$$

In addition we verify immediately that since $H_{1}=H_{0}^{1} \times H_{\text {div }} \subset L^{2} \times\left(L^{2}\right)^{2}=H_{2}$ with dense injection, and that $B_{\mid H_{1} \times H_{1}}$ is symmetric, the solutions of the two problems $\left(P_{1}\right),\left(P_{2}\right)$ are in fact in $H_{0}^{1} \times H_{\text {div }}$ and coincide with the unique solution $(u, \nabla u) \in\left(H_{0}^{1} \cap H^{2}\right) \times\left(H^{1}\right)^{2}$ of Problem $(M)$.

\subsection{The FVbox scheme of lowest order}

In [22], we introduced the following finite-volume scheme for the Poisson problem, called here FVbox scheme for simplicity: find $\left(u_{h}, p_{h}\right) \in P_{n c, 0}^{1} \times R T^{0}$ such that

$$
\left\{\begin{array}{cc}
\left\langle\operatorname{div} p_{h}+f, \mathbb{1}_{K}\right\rangle=0 & \forall K \in \mathcal{T}_{h} \\
\left\langle p_{h}-\nabla u_{h}, \mathbb{1}_{K}\right\rangle=0 & \forall K \in \mathcal{T}_{h} \\
u_{h}=0 & \text { on } \partial \Omega
\end{array}\right.
$$

where $P_{n c, 0}^{1}$ is the nonconforming space of Crouzeix-Raviart with homogeneous boundary conditions, and $R T^{0}$ the div-conforming space of Raviart-Thomas of lowest order defined respectively by

$$
\begin{gathered}
P_{n c, 0}^{1}=\left\{v_{h} / \forall K \in \mathcal{T}_{h}, \quad v_{h \mid K} \in P^{1}(K), v_{h}\right. \text { is continuous at the middle of each edge, } \\
\left.v_{h}=0 \text { at the middle of each edge on } \partial \Omega\right\} \\
R T^{0}=\left\{p_{h} \in H_{\text {div }} / p_{h \mid K} \in R T^{0}(K) \quad \forall K \in \mathcal{T}_{h}\right\}
\end{gathered}
$$

where $R T^{0}(K)$ is the 3 -dimensional space

$$
R T^{0}(K)=\left(P^{0}(K)\right)^{2}+P^{0}(K)\left[\begin{array}{l}
x^{1} \\
x^{2}
\end{array}\right] .
$$

This scheme is in fact a nonconforming Petrov-Galerkin approximation of the mixed formulation $\left(P_{1}\right)$. Consider any regular finite element triangulation $\mathcal{T}_{h}$ (in the usual sense) of the domain $\Omega \subset \mathbb{R}^{2}$. The primal Hilbert space is $H_{1}=X_{1} \times M_{1}=H_{\text {div }} \times H_{0}^{1}$. It is approximated by the space $K_{h}^{1}=X_{1, h} \times M_{1, h}$ with

$$
X_{1, h}=R T^{0}, \quad M_{1, h}=P_{n c, 0}^{1} .
$$

Since $M_{1, h}=P_{n c, 0}^{1} \not \subset M_{1}=H_{0}^{1}$, this is a nonconforming approximation. We check easily that $H_{1, h}=H_{1}+K_{1, h}$ is a Hilbert space, equipped with the mesh-dependent norm $\|(u, p)\|_{1, h}$ defined by

$$
\|(u, p)\|_{1, h}=\left(\|u\|_{h}^{2}+\|p\|_{\operatorname{div}, \Omega}^{2}\right)^{1 / 2} .
$$

where the discrete energy norm on $H_{0}^{1}+P_{n c, 0}^{1}$ is given by

$$
\|u\|_{h}=\left(\sum_{K \in \mathcal{T}_{h}}|\nabla u|_{0, K}^{2}\right)^{1 / 2} .
$$


Recall that this norm is equivalent to $\left(|u|_{0, \Omega}^{2}+\|u\|_{h}^{2}\right)^{1 / 2}$ on $H_{0}^{1}+P_{n c, 0}^{1}$. The norm $\|(u, p)\|_{1, h}$ extends the one of $H_{1}$ to $H_{1, h}$.

Denoting by $P^{0}$ the space of the functions constant in each triangle $K$, the discrete test space is $K_{2, h}=X_{2, h} \times M_{2, h}$, with $X_{2, h}=\left(P^{0}\right)^{2}, \quad M_{2, h}=P^{0}$. We have $K_{2, h} \subset H_{2}$. By symmetry with $H_{1, h}$, we denote $H_{2, h}=H_{2}+K_{2, h}=H_{2}$, and for $(v, q) \in H_{2, h}$, we note

$$
\|(v, q)\|_{2, h}=\left(|v|_{0, \Omega}^{2}+|q|_{0, \Omega}^{2}\right)^{1 / 2}
$$

The FVbox scheme reads now: find $\left(u_{h}, p_{h}\right) \in P_{n c, 0}^{1} \times R T^{0}$ such that for any $\left(v_{h}, q_{h}\right) \in P^{0} \times\left(P^{0}\right)^{2}$

$$
\left(P_{1, h}\right) \begin{cases}\left(\operatorname{div} p_{h}+f, v_{h}\right)_{0, \Omega}=0 & \forall v_{h} \in P^{0} \\ \sum_{K}\left(p_{h}-\nabla u_{h}, q_{h}\right)_{0, K}=0 & \forall q_{h} \in\left(P^{0}\right)^{2} .\end{cases}
$$

The bilinear forms $a_{h}, b_{1, h}, b_{2, h}$ are defined for $u_{h} \in P_{n c, 0}^{1}, v_{h} \in P^{0}, p_{h} \in R T^{0}, q_{h} \in\left(P^{0}\right)^{2}$ by

$$
a_{h}\left(p_{h}, q_{h}\right)=\left(p_{h}, q_{h}\right)_{0, \Omega}, b_{1, h}\left(q_{h}, u_{h}\right)=-\sum_{K}\left(\nabla u_{h}, q_{h}\right)_{0, K}, b_{2, h}\left(p_{h}, v_{h}\right)=\left(\operatorname{div} p_{h}, v_{h}\right)_{0, \Omega}
$$

Defining the continuous linear form $m_{2}$ on $L^{2}$ by

$$
\left\langle m_{2}, v\right\rangle=-(f, v)_{0, \Omega}
$$

Problem $\left(P_{1, h}\right)$ can be rewritten as: find $\left(u_{h}, p_{h}\right) \in P_{n c, 0}^{1} \times R T^{0}$ such that

$$
\begin{cases}a_{h}\left(p_{h}, q_{h}\right)+b_{1, h}\left(q_{h}, u_{h}\right)=0 & \forall q_{h} \in\left(P^{0}\right)^{2} \\ b_{2, h}\left(p_{h}, v_{h}\right)=\left\langle m_{2}, v_{h}\right\rangle & \forall v_{h} \in P^{0}\end{cases}
$$

or equivalently

$$
B_{h}\left[\left(u_{h}, p_{h}\right) ;\left(v_{h}, q_{h}\right)\right]=\left\langle L_{2} ;\left(v_{h}, q_{h}\right)\right\rangle
$$

where the bilinear form $B_{h}$ is

$$
B_{h}\left[\left(u_{h}, p_{h}\right) ;\left(v_{h}, q_{h}\right)\right]=a_{h}\left(p_{h}, q_{h}\right)+b_{1, h}\left(q_{h}, u_{h}\right)+b_{2, h}\left(p_{h}, v_{h}\right)
$$

and the linear continuous form $L_{2}$ on $L^{2} \times\left(L^{2}\right)^{2}$ is

$$
\left\langle L_{2} ;(v, q)\right\rangle=-(f, v)_{0, \Omega} .
$$

Let us denote respectively by $N E, N A, N A_{b}, N A_{i}$ the number of triangles, edges, boundary edges and internal edges. Recall that

$$
\left\{\begin{array}{l}
\operatorname{dim} X_{1, h}=N A, \operatorname{dim} X_{2, h}=2 N E \\
\operatorname{dim} M_{1, h}=N A_{i}, \operatorname{dim} M_{2, h}=N E
\end{array}\right.
$$

and that

$$
3 N E=N A+N A_{i}=2 N A-N A_{b}
$$


which is equivalent to say that

$$
\operatorname{dim} X_{1, h}+\operatorname{dim} M_{1, h}=\operatorname{dim} X_{2, h}+\operatorname{dim} M_{2, h} .
$$

We apply now to this particular case the following general stability and error estimate result for a numerical method having the form $\left(M_{h}\right)$. Suppose we are given four finite-dimensional spaces $X_{1, h}, M_{1, h}, X_{2, h}, M_{2, h}$ approximating the four Hilbert spaces $X_{1}, M_{1}, X_{2}, M_{2}$ with possibly $X_{i, h} \not \subset X_{i}, M_{i, h} \not \subset M_{i}$. In addition, we call $H_{i}=X_{i} \times M_{i}, K_{i, h}=X_{i, h} \times M_{i, h}$, and we suppose that the spaces $H_{i, h}=H_{i}+K_{i, h}$ are Hilbert spaces equipped with norms $\|\cdot\|_{i, h}$ extending to $H_{i, h}$ the norms of $H_{i}$. The approximation of problem $(M)$ is: find $\left(u_{h}, \lambda_{h}\right) \in X_{1, h} \times M_{1, h}$ such that for any $v_{h} \in X_{2, h}, \mu_{h} \in M_{2, h}$ we have

$$
\left(M_{h}\right)\left\{\begin{aligned}
a_{h}\left(u_{h}, v_{h}\right)+b_{1, h}\left(v_{h}, \lambda_{h}\right) & =\left\langle l_{2, h}, v_{h}\right\rangle_{X_{2, h}^{\prime} ; X_{2, h}} \\
b_{2, h}\left(u_{h}, \mu_{h}\right) & =\left\langle m_{2, h}, \mu_{h}\right\rangle_{M_{2, h}^{\prime} ; M_{2, h}}
\end{aligned}\right.
$$

where $l_{2, h}, m_{2, h}$ are approximations of $l_{2}, m_{2}$, and $a_{h}, b_{1, h}, b_{2, h}$ are approximations of the forms $a, b_{1}, b_{2}$. Symmetrically, we introduce problem $\left(M_{h}^{\prime}\right)$ which is the dual problem of $\left(M_{h}\right)$ : find $\left(v_{h}, \mu_{h}\right) \in X_{2, h} \times M_{2, h}$ such that for any $u_{h} \in X_{1, h}, \lambda_{h} \in M_{1, h}$

$$
\left(M_{h}^{\prime}\right) \quad\left\{\begin{aligned}
a_{h}\left(u_{h}, v_{h}\right)+b_{2, h}\left(u_{h}, \mu_{h}\right) & =\left\langle l_{1, h}, u_{h}\right\rangle_{X_{1, h}^{\prime} ; X_{1, h}} \\
b_{1, h}\left(v_{h}, \lambda_{h}\right) & =\left\langle m_{1, h}, \lambda_{h}\right\rangle_{M_{1, h}^{\prime} ; M_{1, h}}
\end{aligned}\right.
$$

where $l_{1, h}, m_{1, h}$ are approximations of $l_{1}, m_{1}$. In [35], Nicolaides describes this kind of problems in the conforming case, when in addition $a_{h}=a, b_{i, h}=b_{i}$. In [7], Bernardi et al. generalize the work of Nicolaides to the conforming case but with possibly $a_{h} \neq a, b_{i, h} \neq b_{i}$. Here, the only difference is that we have to work in the Hilbert spaces $H_{1, h}=H_{1}+K_{1, h}, H_{2, h}=H_{2}+K_{2, h}$, with a possible non-conformity as in [1]. Let us introduce the discrete null spaces $V_{1, h}, V_{2, h}$

$$
\begin{array}{ll}
V_{1, h}=\left\{u_{h} \in X_{1, h} / b_{2, h}\left(u_{h}, \mu_{h}\right)=0\right. & \left.\forall \mu_{h} \in M_{2, h}\right\} \\
V_{2, h}=\left\{v_{h} \in X_{2, h} / b_{1, h}\left(v_{h}, \lambda_{h}\right)=0\right. & \left.\forall \lambda_{h} \in M_{1, h}\right\} .
\end{array}
$$

The discrete counterpart of Theorem 2.1 is

Theorem 2.4. Problem $\left(M_{h}\right)$ has a unique solution $\left(u_{h}, \lambda_{h}\right)$, with continuous dependence (uniform in $h$ ) on the data $\left(l_{2, h}, m_{2, h}\right) \in X_{2, h}^{\prime} \times M_{2, h}^{\prime}$, if and only if the four following conditions hold

$(i)_{h} \quad$ There exists $\bar{\alpha}>0$ independent of $h$ such that for any $v_{h} \in V_{2, h}$

$$
\sup _{u_{h} \in V_{1, h},\left\|u_{h}\right\|_{X_{1, h}} \leq 1} a_{h}\left(u_{h}, v_{h}\right) \geq \bar{\alpha}\left\|v_{h}\right\|_{X_{2, h}}
$$

$(i i)_{h}$

$$
\operatorname{dim} X_{1, h}+\operatorname{dim} M_{1, h}=\operatorname{dim} X_{2, h}+\operatorname{dim} M_{2, h}
$$

$(\text { iii })_{h} \quad$ There exists $\bar{\beta}_{1}>0$ independent of $h$ such that

$$
\sup _{v_{h} \in X_{2, h},\left\|v_{h}\right\|_{X_{2, h}} \leq 1} b_{1, h}\left(v_{h}, \lambda_{h}\right) \geq \bar{\beta}_{1}\left\|\lambda_{h}\right\|_{M_{1, h}}
$$

$\left(\text { iii }_{2}\right)_{h} \quad$ There exists $\bar{\beta}_{2}>0$ independent of $h$ such that

$$
\sup _{u_{h} \in X_{1, h},\left\|u_{h}\right\|_{X_{1, h}} \leq 1} b_{2, h}\left(u_{h}, \mu_{h}\right) \geq \bar{\beta}_{2}\left\|\mu_{h}\right\|_{M_{2, h}} .
$$


We skip the proof, since it follows the same lines as the ones in [35], [7]. We note also, as in Section 2.1, that conditions $(i)_{h}+(i i)_{h}$ on the form $a_{h}$ are equivalent to the dual conditions $\left(i^{\prime}\right)_{h}+(i i)_{h}$, where $\left(i^{\prime}\right)_{h}$ is

$$
\sup _{v_{h} \in V_{2, h},\left\|v_{h}\right\|_{X_{2, h}} \leq 1} a_{h}\left(u_{h}, v_{h}\right) \geq \bar{\alpha}^{\prime}\left\|u_{h}\right\|_{X_{1, h}}
$$

with $\bar{\alpha}^{\prime}$ independent of $h$. Therefore, the set of conditions $(i)_{h},(i i)_{h},\left(i i i_{1}\right)_{h},\left(i i i_{2}\right)_{h}$ is equivalent to the set of conditions $\left(i^{\prime}\right)_{h},(i i)_{h},\left(i i_{1}\right)_{h},\left(i i i_{2}\right)_{h}$ and each of these sets is equivalent to the well-posedness of Problem $\left(M_{h}\right)$ or of Problem $\left(M_{h}^{\prime}\right)$.

We need now the standard error estimate (called second Strang's Lemma), whose proof is similar to the one when only two nonconforming Hilbert spaces $X_{h}, M_{h}$ occur, [8]. We denote

$$
B_{h}\left[\left(u_{h}, \lambda_{h}\right) ;\left(v_{h}, \mu_{h}\right)\right]=a_{h}\left(u_{h}, v_{h}\right)+b_{1, h}\left(v_{h}, \lambda_{h}\right)+b_{2, h}\left(u_{h}, \mu_{h}\right)
$$

and

$$
\left\langle L_{h} ;\left(v_{h}, \mu_{h}\right)\right\rangle=\left\langle l_{2, h} ; v_{h}\right\rangle_{X_{2, h}^{\prime} ; X_{2, h}}+\left\langle m_{2, h} ; \mu_{h}\right\rangle_{M_{2, h}^{\prime} ; M_{2, h}}
$$

we have the following

Theorem 2.5. There exists a constant $C>0$ independent of $h$ such that

$$
\begin{aligned}
&\left\|(u, \lambda)-\left(u_{h}, \lambda_{h}\right)\right\|_{1, h} \leq C\left\{\inf _{\left(\tilde{u}_{h}, \tilde{\lambda}_{h}\right) \in K_{1, h}}\left[\left\|(u, \lambda)-\left(\tilde{u}_{h}, \tilde{\lambda}_{h}\right)\right\|_{1, h}\right]\right. \\
&\left.+\sup _{\left(\tilde{v}_{h}, \tilde{\mu}_{h}\right) \in K_{2, h}} \frac{\left|B_{h}\left[(u, \lambda) ;\left(\tilde{v}_{h}, \tilde{\mu}_{h}\right)\right]-\left\langle L_{h} ;\left(\tilde{v}_{h}, \tilde{\mu}_{h}\right)\right\rangle\right|}{\left\|\left(\tilde{v}_{h}, \tilde{\mu}_{h}\right)\right\|_{2, h}}\right\} .
\end{aligned}
$$

Applying Theorem 2.4 and Theorem 2.5 to discrete problem $\left(P_{1, h}\right)$, we obtain the following proposition, which summarizes in a concize way the result of [22].

Proposition 2.6. (a) Problem $\left(P_{1, h}\right)$ has a unique solution $\left(u_{h}, p_{h}\right) \in P_{n c, 0}^{1} \times R T^{0}$ such that

$$
\left\|u_{h}\right\|_{h}+\left\|p_{h}\right\|_{\operatorname{div}, \Omega} \leq C|f|_{0, \Omega}
$$

(b) For $f \in H^{1}(\Omega)$, the solution $\left(u_{h}, p_{h}\right)$ verifies the error estimate

$$
\left\|u-u_{h}\right\|_{h}+\left\|p-p_{h}\right\|_{\operatorname{div}, \Omega} \leq C h\|f\|_{1, \Omega}
$$

where $C$ stands for a constant independent of $h$.

Proof. The spaces $X_{1, h}, M_{1, h}$ are specified in (32). Moreover, $X_{2, h}=\left(P^{0}\right)^{2}, M_{2, h}=P^{0}$. The bilinear forms $a_{h}$, $b_{1, h}, b_{2, h}, B_{h}$ are given in (37), (40). The forms $l_{2, h}, m_{2, h}$ are given by $\left\langle l_{2, h}, q_{h}\right\rangle=0,\left\langle m_{2, h}, v_{h}\right\rangle=-\left(f, v_{h}\right)_{0, \Omega}$. This gives $\left\|m_{2, h}\right\| \leq|f|_{0, \Omega}$.

(a) According to Theorem 2.4, we check now the conditions $(i)_{h},(i i)_{h},\left(i i i_{1}\right)_{h},\left(i i i_{2}\right)_{h}$ for the forms $a_{h}, b_{1, h}, b_{2, h}$. - $(i)_{h}$ : The spaces $V_{1, h}, V_{2, h}$ are

$$
\begin{array}{cc}
V_{1, h}=\left\{p \in R T^{0} /(\operatorname{div} p, v)_{0, \Omega}=0\right. & \left.\forall v \in P^{0}\right\} \\
V_{2, h}=\left\{q \in\left(P^{0}\right)^{2} / \sum_{K}(q, \nabla u)_{0, K}=0\right. & \left.\forall u \in P_{n c, 0}^{1}\right\} .
\end{array}
$$


The dimensions of these spaces are

$$
\begin{aligned}
& \operatorname{dim} V_{1, h}=\operatorname{dim} X_{1, h}-\operatorname{dim} M_{2, h}=N A-N E \\
& \operatorname{dim} V_{2, h}=\operatorname{dim} X_{2, h}-\operatorname{dim} M_{1, h}=2 N E-N A_{i} .
\end{aligned}
$$

Therefore, due to (44), $\operatorname{dim} V_{1, h}=\operatorname{dim} V_{2, h}$. We prove now that in fact, $V_{1, h}=V_{2, h}$. Suppose given $p \in V_{1, h}$. Then, div $p_{\mid K}=0$ for any $K \in \mathcal{T}_{h}$, therefore $p \in\left(P^{0}\right)^{2}$. Moreover, for any $u \in P_{n c, 0}^{1}$,

$$
\begin{aligned}
\sum_{K}(p, \nabla u)_{0, K} & =-\sum_{K}(\operatorname{div} p, u)_{0, K}+\sum_{K} \int_{\partial K}(p \cdot \nu) u \mathrm{~d} \sigma \\
& =\sum_{K} \int_{\partial K}(p \cdot \nu) u \mathrm{~d} \sigma=-\sum_{a \in A} \int_{a}\left(p \cdot \nu_{a}\right)[u] \mathrm{d} \sigma .
\end{aligned}
$$

where $[u]$ stands for the jump of $u$ in the direction of $\nu_{a}$. (On $\partial \Omega$, we put $[u]=-u_{\text {inside }}$ ). Since $p \in X_{1, h} \subset H_{\text {div }}$, $\left(p \cdot \nu_{a}\right)$ is constant along each edge $a$. Moreover, since $u$ is continuous at the middle of each edge, the average of $[u]$ along $a$ is zero. Therefore $(63)=0$, which gives that $p \in V_{2, h}$. This proves that $V_{1, h} \subset V_{2, h}$. By equality of the dimensions, we obtain

$$
V_{1, h}=V_{2, h}=V_{h} .
$$

Consequently, \|\|$_{\operatorname{div}, \Omega}$ coincides on $V_{h}$ with ||$_{0, \Omega}$. Therefore the condition $\left(i_{h}\right)$ holds because we have for any $q \in V_{h}$

$$
\sup _{p \in V_{h},\|p\|_{\text {div }, \Omega} \leq 1}(p, q)_{0, \Omega}=|q|_{0, \Omega} .
$$

- $(i i)_{h}$ : Condition $(i i)_{h}$ is just (45).

- $\left(i i i_{1}\right)_{h}$ : We have to prove that for any $u \in M_{1, h}$

$$
\sup _{q \in\left(P^{0}\right)^{2},|q|_{0, \Omega} \leq 1} b_{1, h}(q, u) \geq \bar{\beta}_{1}\|u\|_{h} .
$$

Taking $p=\frac{1}{|\nabla u|_{0, \Omega}} \sum_{K} \nabla u_{\mid K} \quad \mathbb{1}_{K}(x)$, we get

$$
\sup _{q \in\left(P^{0}\right)^{2},|q|_{0, \Omega} \leq 1} b_{1, h}(q, u) \geq b_{1, h}(p, u)=\|u\|_{h}
$$

which proves the result.

- $\left(i i i_{2}\right)_{h}$ : This is the well-known inf-sup condition of the standard mixed method of Raviart-Thomas for the couple of spaces $(v, p) \in P^{0} \times R T^{0}$, i.e.

$$
\sup _{p \in R T^{0},\|p\|_{\text {div }, \Omega} \leq 1} b_{2, h}(p, v) \geq \beta_{2}|v|_{0, \Omega} .
$$

We refer to [36] for the proof. 
(b) We prove now the error estimate (57). We deduce from Theorem 2.5 (see (41), (42) for the notation), that:

$$
\begin{aligned}
\left\|u-u_{h}\right\|_{h}+\left\|p-p_{h}\right\|_{\mathrm{div}, \Omega} \leq C\left\{\inf _{\left(\tilde{u}_{h}, \tilde{p}_{h}\right) \in P_{n c, 0}^{1} \times R T^{0}}\left[\left\|u-\tilde{u}_{h}\right\|_{h}+\left\|p-\tilde{p}_{h}\right\|_{\mathrm{div}, \Omega}\right]\right. \\
\left.\quad+\sup _{\left(\tilde{v}_{h}, \tilde{q}_{h}\right) \in P^{0} \times\left(P^{0}\right)^{2}} \frac{\left|B_{h}\left[(u, p) ;\left(\tilde{v}_{h}, \tilde{q}_{h}\right)\right]-\left\langle L_{2} ;\left(\tilde{v}_{h}, \tilde{q}_{h}\right)\right\rangle\right|}{\left\|\left(\tilde{v}_{h} ; \tilde{q}_{h}\right)\right\|_{2, h}}\right\} .
\end{aligned}
$$

Since $p=\nabla u$ and $\operatorname{div} p+f=0$, we have for any $\left(\tilde{v}_{h}, \tilde{q}_{h}\right) \in P^{0} \times\left(P^{0}\right)^{2}$,

$$
B_{h}\left[(u, p) ;\left(\tilde{v}_{h}, \tilde{q}_{h}\right)\right]-\left\langle L_{2} ;\left(\tilde{v}_{h}, \tilde{q}_{h}\right)\right\rangle=\sum_{K}\left(p-\nabla u, \tilde{q}_{h}\right)_{0, K}+\left(\operatorname{div} p, \tilde{v}_{h}\right)_{0, \Omega}+\left(f ; \tilde{v}_{h}\right)_{0, \Omega}=0 .
$$

Thus, the error method vanishes. Finally, the standard estimate of the interpolation error, $[8,36]$

$$
\inf _{\tilde{u}_{h} \in P_{n c, 0}^{1}}\left\|u-\tilde{u}_{h}\right\|_{h}+\inf _{\tilde{p}_{h} \in R T^{0}}\left\|p-\tilde{p}_{h}\right\|_{\operatorname{div}, \Omega} \leq C h\|f\|_{1, \Omega}
$$

yields the result.

\subsection{The dual FVbox scheme}

We consider now discrete problem $\left(P_{2, h}\right)$, dual of $\left(P_{1, h}\right)$, connected with the mixed formulation $\left(P_{2}\right)$ : find $\left(v_{h}, q_{h}\right) \in P^{0} \times\left(P^{0}\right)^{2}$ such that (see (47)) for any $\left(p_{h}, u_{h}\right) \in X_{1, h} \times M_{1, h}$

$$
\left(P_{2, h}\right) \begin{cases}-\sum_{K}\left(\nabla u_{h}, q_{h}\right)_{0, K}=-\left(f, u_{h}\right)_{0, \Omega} & \forall u_{h} \in P_{n c, 0}^{1} \\ \left(p_{h}, q_{h}\right)_{0, \Omega}+\left(\operatorname{div} p_{h}, v_{h}\right)_{0, \Omega}=0 & \forall p_{h} \in R T^{0} .\end{cases}
$$

$\left(P_{2, h}\right)$ can be rewritten, with the help of the form $B_{h}$ : find $\left(v_{h}, q_{h}\right) \in P^{0} \times\left(P^{0}\right)^{2}$ such that for any $\left(u_{h}, p_{h}\right)$ $\in P_{n c, 0}^{1} \times R T^{0}$

$$
B_{h}\left[\left(u_{h}, p_{h}\right) ;\left(v_{h}, q_{h}\right)\right]=\left\langle L_{1, h},\left(u_{h}, p_{h}\right)\right\rangle
$$

where the linear form $L_{1, h} \in H_{1, h}^{\prime}$ is defined for $\left(u_{h}, p_{h}\right) \in H_{1, h}$ by

$$
\left\langle L_{1, h} ;\left(u_{h}, p_{h}\right)\right\rangle=\left\langle m_{1, h}, u_{h}\right\rangle_{M_{1, h}^{\prime} ; M_{1, h}}+\left\langle l_{1, h}, p_{h}\right\rangle_{X_{1, h}^{\prime} ; X_{1, h}}
$$

with $\left\langle m_{1, h}, u_{h}\right\rangle_{M_{1, h}^{\prime} ; M_{1, h}}=-\left(f, u_{h}\right)_{0, \Omega}$ and $\left\langle l_{1, h}, l_{h}\right\rangle_{X_{1, h}^{\prime} ; X_{1, h}}=0$. Recall that $(v, q) \in L^{2} \times\left(L^{2}\right)^{2}$ is the solution of the dual Problem $\left(P_{2}\right)$. We have $(v, q)=(u, p)$ where $(u, p)$ is the solution of the primal Problem $\left(P_{1}\right)$. Symmetrically to Proposition 2.6, we have the following result

Proposition 2.7. (a) Problem $\left(P_{2, h}\right)$ has a unique solution $\left(v_{h}, q_{h}\right) \in P^{0} \times\left(P^{0}\right)^{2}$ such that

$$
\left|v_{h}\right|_{0, \Omega}+\left|q_{h}\right|_{0, \Omega} \leq C|f|_{0, \Omega} .
$$

with $C$ independent of $h$.

(b) This solution satisfies the error estimate

$$
\left|v-v_{h}\right|_{0, \Omega}+\left|q-q_{h}\right|_{0, \Omega} \leq C h|f|_{0, \Omega} .
$$

with $C$ independent of $h$. 
Proof. (a) By Theorem 2.4, a set of necessary and sufficient conditions in order for Problem $\left(P_{2, h}\right)$ to be well posed are conditions $\left(i^{\prime}\right)_{h},(i i)_{h},\left(i i i_{1}\right)_{h},\left(i i_{2}\right)_{h}$. It results from the remark following Theorem 2.4 , that conditions $\left(i^{\prime}\right)_{h},(i i)_{h}$ are equivalent to $(i i i)_{h},(i v)_{h}$, which are true by Proposition 2.6. We conclude by verifying that $\left\|m_{1, h}\right\| \leq C|f|_{0, \Omega}$.

(b) From Theorem 2.5 applied to (47), we have the error estimate

$$
\begin{aligned}
\left|v-v_{h}\right|_{0, \Omega}+\left|q-q_{h}\right|_{0, \Omega} \leq & C\left\{\inf _{\left(\tilde{v}_{h}, \tilde{q}_{h}\right) \in P^{0} \times\left(P^{0}\right)^{2}}\left[\left|v-\tilde{v}_{h}\right|_{0, \Omega}+\left|q-\tilde{q}_{h}\right|_{0, \Omega}\right]\right. \\
& \left.+\sup _{\left(\tilde{u}_{h}, \tilde{p}_{h}\right) \in P_{n c, 0}^{1} \times R T^{0}} \frac{\left|B_{h}\left[\left(\tilde{u}_{h}, \tilde{p}_{h}\right) ;(v, q)\right]-\left\langle L_{1, h} ;\left(\tilde{u}_{h}, \tilde{p}_{h}\right)\right\rangle\right|}{\left\|\left(\tilde{u}_{h} ; \tilde{p}_{h}\right)\right\|_{1, h}}\right\} .
\end{aligned}
$$

Estimating the consistency error, we have for any $\left(\tilde{u}_{h}, \tilde{p}_{h}\right) \in P_{n c, 0}^{1} \times R T^{0}$,

$$
B_{h}\left[\left(\tilde{u}_{h}, \tilde{p}_{h}\right) ;(v, q)\right]-\left\langle L_{1, h},\left(\tilde{u}_{h}, \tilde{p}_{h}\right)\right\rangle=\left(\tilde{p}_{h}, q\right)_{0, \Omega}+\left(\operatorname{div} \tilde{p}_{h}, v\right)_{0, \Omega}-\sum_{K}\left(\nabla \tilde{u}_{h}, q\right)_{0, K}+\left(f, \tilde{u}_{h}\right)_{0, \Omega} .
$$

Because $X_{1, h} \subset H_{\text {div }}$, we have, in view of $(5)_{2}$,

$$
\left(\tilde{p}_{h}, q\right)_{0, \Omega}+\left(\operatorname{div} \tilde{p}_{h}, v\right)_{0, \Omega}=0 \quad \forall \tilde{p}_{h} \in R T^{0} .
$$

Therefore, since $q$ is in fact in $H_{\text {div }}$,

$$
\begin{aligned}
-\sum_{K}\left(\nabla \tilde{u}_{h}, q\right)_{0, K}+\left(f, \tilde{u}_{h}\right)_{0, \Omega} & =\sum_{K}\left(\tilde{u}_{h}, \operatorname{div} q\right)_{0, K}+\left(f, \tilde{u}_{h}\right)_{0, \Omega}-\sum_{K} \int_{\partial K} \tilde{u}_{h}(q \cdot \nu) \mathrm{d} \sigma \\
& =-\sum_{K} \sum_{e \in \partial K} \int_{e} \tilde{u}_{h}(q \cdot \nu) \mathrm{d} \sigma+\left(\tilde{u}_{h}, \operatorname{div} q+f\right)_{0, \Omega} .
\end{aligned}
$$

The second term in (79) is zero in view of $\left(P_{2}\right)_{1}$. The first term in (79) is rewritten as $\left(A_{i}\right.$ is the set of the internal edges, and $A_{b}$ the set of the boundary edges)

$$
\sum_{a \in A_{i}} \int_{a}\left[\tilde{u}_{h}\right] \quad\left(q \cdot \nu_{a}\right) \mathrm{d} \sigma-\sum_{a \in A_{b}} \int_{a} \tilde{u}_{h}\left(q \cdot \nu_{a}\right) \mathrm{d} \sigma
$$

By the continuity of $\tilde{u}_{h}$ at the middle of each edge, and since $\tilde{u}_{h}\left(x_{a}\right)=0$ for $x_{a}$ the middle of an edge $a$ on $\partial \Omega$, we have (recall that $\tilde{u}_{h \mid a}$ is affine)

$$
\int_{a \in A_{i}}\left[\tilde{u}_{h}\right] \mathrm{d} \sigma=0 ; \int_{a \in A_{b}} \tilde{u}_{h} \mathrm{~d} \sigma=0 .
$$

Therefore, denoting by $\tilde{u}_{h, i}, i=1,2$, the traces of $\tilde{u}_{h}$ on each side of the edge $a$, and by $\Pi_{a} \tilde{u}_{h}$ the common mean value of $\tilde{u}_{h, 1}, \tilde{u}_{h, 2}$ along the edge $a$, we obtain that $(80)$ can be rewritten as

$$
\sum_{a \in A_{i}}\left[\int_{a}\left(\tilde{u}_{h, 2}-\Pi_{a} \tilde{u}_{h}\right)\left(q \cdot \nu_{a}\right) \mathrm{d} \sigma-\int_{a}\left(\tilde{u}_{h, 1}-\Pi_{a} \tilde{u}_{h}\right)\left(q \cdot \nu_{a}\right) \mathrm{d} \sigma\right]-\sum_{a \in A_{b}} \int_{a}\left(\tilde{u}_{h, 1}-\Pi_{a} \tilde{u}_{h}\right)\left(q \cdot \nu_{a}\right) \mathrm{d} \sigma
$$

Thus, by Lemma 3 of [24], we get for each internal edge $a \in A_{i}$

$$
\left|\int_{a \in A_{i}}\left[\tilde{u}_{h}\right]\left(q \cdot \nu_{a}\right) \mathrm{d} \sigma\right| \leq C h\left[|q|_{1, K_{1}}\left|\tilde{u}_{h}\right|_{1, K_{1}}+|q|_{1, K_{2}}\left|\tilde{u}_{h}\right|_{1, K_{2}}\right]
$$


and for each boundary edge $a \in A_{b}$

$$
\left|\int_{a \in A_{b}} \tilde{u}_{h}\left(q \cdot \nu_{a}\right) \mathrm{d} \sigma\right| \leq C h|q|_{1, K_{1}}\left|\tilde{u}_{h}\right|_{1, K_{1}}
$$

where $a$ is oriented from $K_{1}$ towards $K_{2}$, and $C$ is a constant independent of $h$. We deduce finally from (83), (84) by the Cauchy-Schwarz inequality the estimate

$$
\left|B_{h}\left[\left(\tilde{u}_{h}, \tilde{p}_{h}\right) ;(v, q)\right]-\left\langle L_{1, h} ;\left(\tilde{u}_{h}, \tilde{p}_{h}\right)\right\rangle\right| \leq 3 C h|q|_{1, \Omega}\left\|\tilde{u}_{h}\right\|_{h} .
$$

which gives the following estimate of the consistency error in (76)

$$
\sup _{\left(\tilde{u}_{h}, \tilde{p}_{h}\right) \in P_{n c, 0}^{1} \times R T^{0}} \frac{\left|B_{h}\left[\left(\tilde{u}_{h}, \tilde{p}_{h}\right) ;(v, q)\right]-\left\langle L_{1, h} ;\left(\tilde{u}_{h}, \tilde{p}_{h}\right)\right\rangle\right|}{\left.\| \tilde{u}_{h}, \tilde{p}_{h}\right) \|_{1, h}} \leq 3 C h|q|_{1, \Omega} .
$$

For the interpolation error, we have the two standard estimates

$$
\inf _{\tilde{v}_{h} \in P^{0}}\left|v-\tilde{v}_{h}\right|_{0, \Omega} \leq C h|v|_{1, \Omega} ; \inf _{\tilde{q}_{h} \in\left(P^{0}\right)^{2}}\left|q-\tilde{q}_{h}\right|_{0, \Omega} \leq C h|q|_{1, \Omega}
$$

Since $|v|_{1, \Omega} \leq\|v\|_{1, \Omega} \leq|f|_{0, \Omega},|q|_{1, \Omega} \leq|f|_{0, \Omega}$ we get the result.

\subsection{Second order error estimate}

In this section, we derive an $O\left(h^{2}\right)$ error estimate in the $L^{2}$ norm for $\left|u-u_{h}\right|_{0, \Omega}$. Such an estimate makes use in the standard conforming finite element method of the Aubin-Nitsche argument. Recall that this argument leads to an estimate like

$$
\left|u-u_{h}\right|_{0, \Omega} \leq C\left\|u-u_{h}\right\|_{1, \Omega}\left\|v-v_{h}\right\|_{1, \Omega}
$$

where $v, v_{h}$ are the continuous solution and discrete approximation of an adjoint problem. Therefore a first order error estimate in the $H^{1}$ norm for $u$ and $v$ yields the second order convergence of $\left|u-u_{h}\right|_{0, \Omega}$. Here, we follow the same principle.

Theorem 2.8. The solution $u_{h} \in P_{n c, 0}^{1}$ of the FVbox scheme $\left(P_{1, h}\right)$ satisfies the second order error estimate

$$
\left|u-u_{h}\right|_{0, \Omega} \leq C h^{2}\|f\|_{1, \Omega}
$$

where $C$ is independent of $h$.

Proof. We follow the same principle as in the proof of the second order error estimate for the standard nonconforming FEM method in $P_{n c, 0}^{1},[8]$.

We start from

$$
\left|u-u_{h}\right|_{0, \Omega}=\sup _{g \in L^{2}, g \neq 0} \frac{\left(u-u_{h}, g\right)_{0, \Omega}}{|g|_{0, \Omega}}
$$

To each $g \in L^{2}(\Omega)$, corresponds the solution $\left(v^{g}, q^{g}\right) \in L^{2} \times\left(L^{2}\right)^{2}$ of Problem $\left(P_{2}\right)_{g}$

$$
B_{h}\left[(u, p) ;\left(v^{g}, q^{g}\right)\right]=-(g, u)_{0, \Omega} \quad \forall(u, p) \in H_{0}^{1} \times H_{\mathrm{div}}
$$

which is also the unique solution in $H_{0}^{1} \times H_{\text {div }}$ of

$$
B_{h}\left[\left(v^{g}, q^{g}\right) ;(v, q)\right]=-(g, v)_{0, \Omega} \quad \forall(v, q) \in L^{2} \times\left(L^{2}\right)^{2} .
$$


We denote by $\left(v_{h}^{g}, q_{h}^{g}\right) \in P^{0} \times\left(P^{0}\right)^{2}$ the discrete solution of Problem $\left(P_{2, h}\right)_{g}$

$$
B_{h}\left[(\tilde{u}, \tilde{p}) ;\left(v_{h}^{g}, q_{h}^{g}\right)\right]=-(g, \tilde{u})_{0, \Omega} \quad \forall(\tilde{u}, \tilde{p}) \in P_{n c, 0}^{1} \times R T^{0} .
$$

In addition, recall that $(u, p) \in H_{0}^{1} \times H_{\text {div }}$ and $\left(u_{h}, p_{h}\right) \in P_{n c, 0}^{1} \times R T^{0}$ are solutions of the continuous and FVbox problems (4), (36).

For any $g \in L^{2}$, we have

$$
\begin{aligned}
-\left(u-u_{h}, g\right)_{0, \Omega}= & B_{h}\left[(u, p) ;\left(v^{g}, q^{g}\right)\right]-B_{h}\left[\left(u_{h}, p_{h}\right) ;\left(v_{h}^{g}, q_{h}^{g}\right)\right] \\
= & B_{h}\left[(u, p)-\left(u_{h}, p_{h}\right) ;\left(v^{g}, q^{g}\right)-\left(v_{h}^{g}, q_{h}^{g}\right)\right](I) \\
& +B_{h}\left[\left(u_{h}, p_{h}\right) ;\left(v^{g}, q^{g}\right)-\left(v_{h}^{g}, q_{h}^{g}\right)\right] \quad(I I) \\
& +B_{h}\left[(u, p)-\left(u_{h}, p_{h}\right) ;\left(v_{h}^{g}, q_{h}^{g}\right)\right] . \quad(I I I)
\end{aligned}
$$

Since $\left(v_{h}^{g}, q_{h}^{g}\right) \in P^{0} \times\left(P^{0}\right)^{2} \subset L^{2} \times\left(L^{2}\right)^{2}$, we have $(I I I)=0$, by subtracting (36) from (4). Moreover, we deduce from the two standard error estimates $(57),(75)$ that

$$
\begin{aligned}
|(I)| & \leq C\left\|(u, p)-\left(u_{h}, p_{h}\right)\right\|_{1, h}\left\|\left(v^{g}, q^{g}\right)-\left(v_{h}^{g}, q_{h}^{g}\right)\right\|_{2, h} \\
& \leq C h^{2}|g|_{0, \Omega}\|f\|_{1, \Omega} .
\end{aligned}
$$

Finally, we have for $(I I)$,

$$
\begin{aligned}
-(I I) & =B_{h}\left[(u, p)-\left(u_{h}, p_{h}\right),\left(v^{g}, q^{g}\right)\right]+\left(g ; u-u_{h}\right) \\
& =\sum_{K}\left(p-p_{h}-\nabla\left(u-u_{h}\right), q^{g}\right)_{0, K}+\left(\operatorname{div}\left(p-p_{h}\right), v^{g}\right)_{0, \Omega}+\left(g, u-u_{h}\right)_{0, \Omega} .
\end{aligned}
$$

Since $\sum_{K}\left(p-p_{h}, q^{g}\right)_{0, K}=\left(p-p_{h}, \nabla v^{g}\right)_{0, \Omega}=-\left(\operatorname{div}\left(p-p_{h}\right), v^{g}\right)_{0, \Omega}$, we obtain

$$
-(I I)=-\sum_{K}\left(\nabla\left(u-u_{h}\right), q^{g}\right)_{0, K}+\left(g, u-u_{h}\right)_{0, \Omega}
$$

and by the Green formula on each triangle $K$

$$
-(I I)=-\sum_{K} \int_{\partial K}\left(u-u_{h}\right)\left(q^{g} \cdot \nu\right) \mathrm{d} \sigma=\sum_{a \in \mathcal{A}} \int_{a}\left[u-u_{h}\right]\left(q^{g} \cdot \nu_{a}\right) \mathrm{d} \sigma .
$$

Calling $\Pi_{a}$ the averaging operator on the edge $a$, we have $\left[u-u_{h}\right]=\left[u-u_{h}\right]-\Pi_{a}\left(\left[u-u_{h}\right]\right)$, since $[u]=0$ and $\Pi_{a}\left[u_{h}\right]=0$. Therefore

$$
\begin{aligned}
|(I I)| & \leq \sum_{a \in \mathcal{A}} \int_{a}\left|\left\{\left[u-u_{h}\right]-\Pi_{a}\left(\left[u-u_{h}\right]\right)\right\}\left(q^{g} \cdot \nu_{a}\right)\right| \mathrm{d} \sigma \\
& \leq 3 C h \sum_{K}\left|u-u_{h}\right|_{1, K}\left|q^{g}\right|_{1, K} \leq 3 C h\left\|u-u_{h}\right\|_{h}\left|q^{g}\right|_{1, \Omega} \\
& \leq C^{\prime} h^{2}\|f\|_{1, \Omega}\left|v^{g}\right|_{2, \Omega} \leq C^{\prime} h^{2}\|f\|_{1, \Omega}|g|_{0, \Omega} .
\end{aligned}
$$

Finally, we get the estimate

$$
\begin{aligned}
\left|\left(u-u_{h}, g\right)_{0, \Omega}\right| & \leq|(I)|+|(I I)|+|(I I I)| \\
& \leq C h^{2}\|f\|_{1, \Omega}|g|_{0, \Omega} .
\end{aligned}
$$

Dividing (95) by $|g|_{0, \Omega}$ and taking the suppremum on $g \neq 0$, we get the desired result. 


\section{FURTHER REMARKS}

\subsection{Comparison with the nonconforming method}

The standard nonconforming method for (3) is: find $\tilde{u}_{h} \in P_{n c, 0}^{1}$ such that

$$
\sum_{K}\left(\nabla \tilde{u}_{h}, \nabla v_{h}\right)_{0, K}=\left(f, v_{h}\right)_{0, \Omega} \quad \forall v_{h} \in P_{n c, 0}^{1} .
$$

For (97), the two following error estimates hold, [8]

$$
\left\|u-\tilde{u}_{h}\right\|_{h} \leq C h|f|_{0, \Omega} \quad\left|u-\tilde{u}_{h}\right|_{0, \Omega} \leq C h^{2}|f|_{0, \Omega} .
$$

Denoting by $\Pi$ the orthogonal projector form $L^{2}$ onto $P^{0}$, we have that $u_{h}$ in $\left(P_{1, h}\right)$ is the following modification of (97).

Proposition 3.1. The function $u_{h}$ in $\left(P_{1, h}\right)$ is the solution of the scheme: find $u_{h} \in P_{n c, 0}^{1}$ such that

$$
\sum_{K}\left(\nabla u_{h}, \nabla v_{h}\right)_{0, K}=\left(\Pi f, v_{h}\right)_{0, \Omega} \quad \forall v_{h} \in P_{n c, 0}^{1} .
$$

Proof. Taking $q_{h}=\nabla v_{h}$ in $\left(P_{1, h}\right)$ in $(36)$, and taking in account that $\operatorname{div} p_{h \mid K}$ is constant, we obtain

$$
\begin{aligned}
\sum_{K}\left(\nabla u_{h}, \nabla v_{h}\right)_{0, K} & =-\sum_{K}\left(\operatorname{div} p_{h}, v_{h}\right)_{0, K}-\sum_{a} \int_{a}\left(p_{h} . \nu\right)\left[v_{h}\right] \mathrm{d} \sigma=-\sum_{K}\left(\operatorname{div} p_{h}, \Pi v_{h}\right)_{0, K} \\
& =\left(f, \Pi v_{h}\right)_{0, \Omega}=\left(\Pi f, v_{h}\right)_{0, \Omega} .
\end{aligned}
$$

Note that we can deduce from Proposition 3.1 that $\left\|u-u_{h}\right\|_{h} \leq C h|f|_{0, \Omega}$, which is better than (57). (The bound $\|f\|_{1, \Omega}$ is due in (57) to the interpolation error for $p_{h}$.) However, the second order error estimate deduced from (99) is identical to the one in Theorem 2.8 .

\subsection{Comparison with the mixed method}

The question arises naturally about the link between the FVbox solution $\left(u_{h}, p_{h}\right)$ of $\left(P_{1, h}\right)$ and the solution $\left(\bar{u}_{h}, \bar{p}_{h}\right)$ of the standard mixed method of Raviart-Thomas. Recall that both $p_{h}, \bar{p}_{h}$ belong to the same space, namely the space $R T^{0}$. In addition, calling $\Pi v_{h}$ the orthogonal projection onto the space $P^{0}$ of a function $v_{h} \in P_{n c, 0}^{1}$, it is interesting to compare $\bar{u}_{h}$ and $\Pi u_{h}$. We have

Proposition 3.2. (i) $p_{h}=\bar{p}_{h}$. In other words, $p_{h}$ coincides with the approximate gradient of $u$ provided by the mixed method.

(ii) We have

$$
\bar{u}_{h}=\Pi u_{h}+\frac{1}{4} \sum_{K}(\Pi f)_{K} \rho_{K}^{2} \mathbb{1}_{K}
$$

where $\rho_{K}$ is the gyration radius of $K$ defined by $|K| \rho_{K}^{2}=\left|\overrightarrow{g_{K}} \vec{x}\right|_{0, K}^{2}$ and $g_{K}$ is the barycentre of $K$.

Proof. (i) The mixed scheme is: find $\left(\bar{u}_{h}, \bar{p}_{h}\right) \in P^{0} \times R T^{0}$ such that:

$$
\begin{cases}\left(\operatorname{div} \bar{p}_{h}+f, \tilde{v}\right)_{0, \Omega}=0 & \forall \tilde{v} \in P^{0} \\ \left(\bar{p}_{h}, \tilde{q}\right)_{0, \Omega}+\left(\bar{u}_{h}, \operatorname{div} \tilde{q}\right)_{0, \Omega}=0 & \forall \tilde{q} \in R T^{0}\end{cases}
$$


whereas the FVbox scheme is: find $\left(u_{h}, p_{h}\right) \in P_{n c, 0}^{1} \times R T^{0}$ such that:

$$
\begin{cases}\left(\operatorname{div} p_{h}+f, \tilde{v}\right)_{0, \Omega}=0 & \forall \tilde{v} \in P^{0} \\ \left(p_{h}, q\right)_{0, \Omega}-\sum_{K}\left(\nabla u_{h}, q\right)_{0, K}=0 & \forall q \in\left(P^{0}\right)^{2} .\end{cases}
$$

Defining $\hat{p} \in R T^{0}$ by $\hat{p}=p_{h}-\bar{p}_{h}$, we get by subtracting $(103)_{1}$, from $(104)_{1}$,

$$
(\operatorname{div} \hat{p}, \tilde{v})_{0, \Omega}=0 \quad \forall \tilde{v} \in P^{0}
$$

therefore $\hat{p} \in V_{h}$, the common null space of the bilinear forms $b_{1, h}, b_{2, h}$, (see (64)). Recall that this space is

$$
\begin{aligned}
V_{h} & =\left\{p_{h} \in R T^{0} /\left(\operatorname{div} p_{h}, v_{h}\right)_{0, \Omega}=0, \quad \forall v_{h} \in P^{0}\right\} \\
& =\left\{q_{h} \in\left(P^{0}\right)^{2} / \sum_{K}\left(q_{h}, \nabla u_{h}\right)_{0, K}=0, \quad \forall u_{h} \in P_{n c, 0}^{1}\right\} \\
& =R T^{0} \cap\left(P^{0}\right)^{2} .
\end{aligned}
$$

Therefore, subtracting $(103)_{2}$ from $(104)_{2}$, we get for any $q \in V_{h}$ :

$$
(\hat{p}, q)_{0, \Omega}=\sum_{K}\left(\nabla u_{h}, q\right)_{0, K}+\left(\bar{u}_{h}, \operatorname{div} q\right)_{0, \Omega}=0 .
$$

Taking $q=\hat{p}$, we obtain $\hat{p}=0$, that is $p_{h}=\bar{p}_{h}$.

(ii) For any $\tilde{q} \in R T^{0}$, we estimate now $\left(\Pi u_{h}, \operatorname{div} \tilde{q}\right)$. We have

$$
\left(\Pi u_{h}, \operatorname{div} \tilde{q}\right)_{0, \Omega}=\left(u_{h}, \operatorname{div} \tilde{q}\right)_{0, \Omega}=-\left(\nabla u_{h}, \tilde{q}\right)_{0, \Omega}-\sum_{a \in \mathcal{A}_{i}} \int_{a}\left[u_{h}\right]\left(\tilde{q} \cdot \nu_{a}\right)
$$

The second term in the right hand side of (107) vanishes $\left(\tilde{q} \cdot \nu_{a}\right.$ is constant on $\left.a\right)$. Now, denoting $g_{K}$ the barycentre of the triangle $K$, we have the two following identities

$$
\begin{gathered}
\tilde{q}=\Pi \tilde{q}+\frac{1}{2} \sum_{K}(\operatorname{div} \tilde{q})_{K} \overrightarrow{g_{K} x} \mathbb{1}_{K} \\
\nabla u_{h}=p_{h}-\frac{1}{2} \sum_{K}\left(\operatorname{div} p_{h}\right)_{K} \overrightarrow{g_{K} x} \mathbb{1}_{K}
\end{gathered}
$$

We deduce from (108), (109) that the first term in the right hand side of (107) is

$$
-\left(\nabla u_{h}, \tilde{q}\right)_{0, \Omega}=-\left(p_{h}, \tilde{q}\right)_{0, \Omega}+\frac{1}{4} \sum_{K}\left(\operatorname{div} p_{h}\right)_{K}(\operatorname{div} \tilde{q})_{K} \quad\left|\overrightarrow{g_{K}} \vec{x}\right|_{0, K}^{2} .
$$

Defining the gyration radius $\rho_{K}$ of the triangle $K$ by $|K| \rho_{K}^{2}=\left|\overrightarrow{g_{K}} \vec{x}\right|_{0, K}^{2},(c f .[5])$, we deduce from (107), (110) and from $\left(\operatorname{div} p_{h}\right)_{K}=(\Pi f)_{K}$ that

$$
\left(\Pi u_{h}+\frac{1}{4} \sum_{K}(\Pi f)_{K} \rho_{K}^{2} \mathbb{1}_{K}, \operatorname{div} \tilde{q}\right)_{0, \Omega}+\left(p_{h}, \tilde{q}\right)_{0, \Omega}=0 \quad \forall \tilde{q} \in R T^{0} .
$$


It results from (103) that

$$
\bar{u}_{h}=\Pi u_{h}+\frac{1}{4} \sum_{K}(\Pi f)_{K} \rho_{K}^{2} \mathbb{1}_{K}
$$

We deduce easily from (112) the estimate $\left|\Pi u_{h}-\bar{u}_{h}\right|_{0, \Omega} \leq C h^{2}|f|_{0, \Omega}$.

\subsection{Comparison with the Lagrange multipliers method}

In [2], Arnold and Brezzi describe an interpretation of the mixed method of Raviart and Thomas based on the relaxation of the divergence conformity of the $R T^{0}(K)$ element by means of a Lagrange multiplier on each edge. See also [12]. Let us describe briefly the method. We need only the lowest order version in the sequel. We call $\mathcal{R} \mathcal{T}_{0}$ the finite element space constructed as

$$
\mathcal{R} \mathcal{T}_{0}=\left\{p_{h}(x)=\sum_{K} p_{h \mid K}(x) \mathbb{1}_{K}(x) / p_{h \mid K} \in R T^{0}(K)\right\}
$$

We have $\operatorname{dim}\left(\mathcal{R} \mathcal{T}_{0}\right)=3 N E$, and $R T^{0}=\mathcal{R} \mathcal{T}_{0} \cap H_{\text {div }}$. The space of Lagrange multipliers is the set of functions defined only on the edges $A$. The lowest order version is simply the functions constant on each edge $a \in A$

$$
\mathcal{S}_{0}^{0}=\left\{\lambda_{h}(x)=\lambda_{a}, x \in a, a \in A_{i}\right\}
$$

The scheme is: find $\left(\bar{u}_{h}, \bar{p}_{h}, \bar{\lambda}_{h}\right) \in P^{0} \times \mathcal{R} \mathcal{T}_{0} \times \mathcal{S}_{0}^{0}$ such that for any $(\tilde{v}, \tilde{q}, \tilde{\mu}) \in P^{0} \times \mathcal{R} \mathcal{T}_{0} \times \mathcal{S}_{0}^{0}$

$$
\left\{\begin{array}{l}
\sum_{K}\left(\operatorname{div} \bar{p}_{h}+f, \tilde{v}\right)_{0, K}=0 \\
\left(\bar{p}_{h}, \tilde{q}\right)_{0, \Omega}+\sum_{K}\left[\left(\bar{u}_{h}, \operatorname{div} \tilde{q}\right)_{0, K}-\int_{\partial K} \bar{\lambda}_{h}(\tilde{q} \cdot \nu) \mathrm{d} \sigma\right]=0 \\
\sum_{K} \int_{\partial K} \tilde{\mu}\left(\bar{p}_{h} \cdot \nu\right)=0
\end{array}\right.
$$

System (115) has a unique solution $\left(\bar{u}_{h}, \bar{p}_{h}, \bar{\lambda}_{h}\right) \in P^{0} \times \mathcal{R} \mathcal{T}_{0} \times \mathcal{S}_{0}^{0}$. Moreover $\left(\bar{u}_{h}, \bar{p}_{h}\right) \in P^{0} \times R T^{0}$ is the standard mixed solution of (103). We may now lift the function $\left(\bar{u}_{h}, \bar{\lambda}_{h}\right)$ in an approximation $\hat{u}_{h}$ lying in a space of higher precision than $P^{0}$. Two choices are presented in [2]. The first one is simply to take $\hat{u}_{h} \in P_{n c}^{1}$ such that

$$
\Pi_{a} \hat{u}_{h}=\lambda_{h, a} \quad \forall a \in A_{i}
$$

The second one is to take $\hat{u}_{h} \in N=P_{n c}^{1}+B^{3}$ where $B^{3}$ is the "bubble" space

$$
B^{3}=\left\{u_{h} / u_{h \mid K} \in \operatorname{Vect}\left(\lambda_{1}(x) \lambda_{2}(x) \lambda_{3}(x)\right), x \in K\right\} .
$$

The lifting $\hat{u}_{h}$ is uniquely defined by

$$
\Pi_{K} \hat{u}_{h}=\bar{u}_{h} ; \Pi_{a} \hat{u}_{h}=\lambda_{h, a}
$$

$\Pi_{a}$ and $\Pi_{K}$ are the orthogonal projections onto the constants in $L^{2}(K)$ and $L^{2}(a)$. In fact, it can be proved, that the lowest order version of the FVbox scheme coincides with the lifting (116).

Proposition 3.3. The solution $\left(u_{h}, p_{h}\right)$ of the FVbox scheme $\left(P_{1, h}\right)$ coincides with the $\left(\hat{u}_{h}, \bar{p}_{h}\right)$ solution of scheme (115), where $\hat{u}_{h}$ is the $P_{n c}^{1}$ interpolation (116). 
Proof. Recall that $p_{h}=\bar{p}_{h}$, where $\bar{p}_{h} \in R T^{0}$ is the solution of the mixed method. For any $\tilde{q} \in \mathcal{R} \mathcal{T}_{0}, \tilde{q} \cdot \nu$ is constant along each edge $e \in \partial K$. Therefore it is sufficient to prove that $\left(u_{h}, p_{h}\right)$ verifies, for any $\tilde{q} \in \mathcal{R} \mathcal{T}_{0}$

$$
\left(p_{h}, \tilde{q}\right)_{0, \Omega}+\sum_{K}\left[\left(\bar{u}_{h}, \operatorname{div} \tilde{q}\right)_{0, K}-\int_{\partial K} u_{h}(\tilde{q} \cdot \nu) \mathrm{d} \sigma\right]=0 .
$$

Since $\tilde{q}(x)_{K}=(\Pi \tilde{q})_{K}+\frac{1}{2}(\operatorname{div} \tilde{q})_{K} \overrightarrow{g_{K} x}$, we have

$$
\begin{aligned}
\left(p_{h}, \tilde{q}\right)_{0, \Omega} & =\left(p_{h}, \Pi \tilde{q}\right)_{0, \Omega}+\frac{1}{2}\left(p_{h}, \sum_{K}(\operatorname{div} \tilde{q})_{K} \quad \overrightarrow{g_{K}} \vec{x} \mathbb{1}_{K}\right)_{0, \Omega} \\
& =\sum_{K}\left(\nabla u_{h}, \Pi \tilde{q}\right)_{0, K}+\frac{1}{2}\left(p_{h}, \sum_{K}(\operatorname{div} \tilde{q})_{K} \overrightarrow{g_{K} \vec{x}} \mathbb{1}_{K}\right)_{0, \Omega}
\end{aligned}
$$

Replacing $p_{h \mid K}$ by its value $p_{h \mid K}=\left(\Pi p_{h}\right)_{K}-\frac{1}{2}(\Pi f)_{K} \overrightarrow{g_{K}} \vec{x}$ we find

$$
\begin{aligned}
\left(p_{h}, \tilde{q}\right)_{0, \Omega} & =\sum_{K}\left(\nabla u_{h}, \tilde{q}\right)_{0, K}-\frac{1}{4} \sum_{K}|K|(\Pi f)_{K}(\operatorname{div} \tilde{q})_{K} \rho_{K}^{2} \\
& =-\sum_{K}\left[\left(u_{h}, \operatorname{div} \tilde{q}\right)_{0, K}-\int_{\partial K} u_{h}(\tilde{q} \cdot \nu) \mathrm{d} \sigma\right]-\frac{1}{4} \sum_{K}|K|(\Pi f)_{K}(\operatorname{div} \tilde{q})_{K} \rho_{K}^{2} \\
& =-\sum_{K}\left[\left(\left(\Pi u_{h}\right)_{K}, \operatorname{div} \tilde{q}\right)_{0, K}-\int_{\partial K} u_{h}(\tilde{q} \cdot \nu) \mathrm{d} \sigma\right]-\frac{1}{4} \sum_{K}|K|(\Pi f)_{K}(\operatorname{div} \tilde{q})_{K} \rho_{K}^{2} .
\end{aligned}
$$

Using (102) we obtain finally

$$
\left(p_{h}, \tilde{q}\right)_{0, \Omega}=-\sum_{K}\left[\left(\bar{u}_{h}, \operatorname{div} \tilde{q}\right)_{0, K}-\int_{\partial K} u_{h}(\tilde{q} \cdot \nu) \mathrm{d} \sigma\right]
$$

which is the desired result.

\subsection{Final remark}

In this paper, we have emphasized the mixed Petrov-Galerkin structure of the scheme introduced in [22]. This structure allows to deduce the numerical analysis of this scheme from the standard theory. This approach may also be applied to schemes of the same kind with different choices of the four spaces $X_{1, h}, X_{2, h}, M_{1, h}, M_{2, h}$. Another possibility is to eliminate $p_{h}$ locally in each cell, and to obtain the error estimates for $u_{h}$ from the scheme (99). The error estimate for $p_{h}$ is deduced afterwards, as in [22], from the local representation formula

$$
p_{h, K}=\nabla u_{h, K}-|K|(\Pi f)_{K} P_{K}(x)
$$

where $P_{K}(x)=\frac{1}{2|K|} \overrightarrow{g_{K}} \vec{x}$.

Let us mention finally that the method given by Marini, [33], for computing the mixed solution $\left(\bar{u}_{h}, \bar{p}_{h}\right)$ of Raviart-Thomas (103), amounts precisely to compute the solution $u_{h}$ in (99) and to express afterwards $p_{h}$ by (121). The key point of the present paper is that this a posteriori interpretation is nothing but the FVbox scheme (36). This scheme has the advantage to give a direct access to an approximation $\left(u_{h}, p_{h}\right)$, affine per cell, without any reference to the mixed method.

Acknowledgements. The author acknowledges friendly J-J. Chattot, B. Courbet, F. Dubois, A. Debussche, V. Girault, J. Laminie, J-.F. Maitre, for stimulating discussions about box schemes, mixed methods and least squares methods. He acknowledges also an anonymous referee for helpful remarks. Note that J.-F. Maître devised also recently the scheme (36). 


\section{REFERENCES}

[1] B. Achchab, A. Agouzal, J. Baranger and J-F. Maître, Estimateur d'erreur a posteriori hiérarchique. Application aux éléments finis mixtes. Numer. Math. 80 (1998) 159-179.

[2] D.N. Arnold and F. Brezzi, Mixed and non-conforming finite elements methods: implementation, postprocessing and error estimates. RAIRO - Modél. Math. Anal. Numér. 19 (1985) 7-32.

[3] I. Babuška, Error-Bounds for Finite Elements Method. Numer. Math. 16 (1971) 322-333.

[4] R.E. Bank and D.J. Rose, Some error estimates for the box method. SIAM J. Numer. Anal. 24 (1987) $777-787$.

[5] J. Baranger, J.F. Maître and F. Oudin, Connection between finite volume and mixed finite element methods. RAIRO - Modél. Math. Anal. Numér. 30 (1996) 445-465.

[6] C. Bernardi, C. Canuto and Y. Maday, Un problème variationnel abstrait. Application à une méthode de collocation pour les équations de Stokes. C. R. Acad. Sci. Paris, t.303, Série I 19 (1986) 971-974.

[7] C. Bernardi, C. Canuto and Y. Maday, Generalized inf-sup conditions for Chebyshev spectral approximation of the Stokes problem. SIAM J. Numer. Anal. 25 (1988) 1237-1271.

[8] D. Braess, Finite Elements. Cambridge Univ. Press (1997).

[9] S.C. Brenner and L.R. Scott, The mathematical theory of finite element methods. Texts Appl. Math. 15 (1994) Springer, New-York.

[10] F. Brezzi, On the existence, uniqueness and approximation of saddle-point problems, arising from lagrangian multipliers. RAIRO 8 (1974) R-2, 129-151.

[11] F. Brezzi and M. Fortin, Mixed and Hybrid Finite Element Methods. Springer Series Comp. Math. 15, Springer Verlag, New-York (1991).

[12] F. Brezzi, J. Douglas and L.D. Marini, Two families of Mixed Finite Element for second order elliptic problems. Numer. Math. 47 (1985) 217-235.

[13] Z. Cai, J. Mandel and S. McCormick, The finite volume element method for diffusion equations on general triangulations. SIAM J. Numer. Anal. 28 (1991) 392-402.

[14] F. Casier, H. Deconninck and C. Hirsch, A class of central bidiagonal schemes with implicit boundary conditions for the solution of Euler's equations. AIAA-83-0126 (1983).

[15] J.J. Chattot, Box-schemes for First Order Partial Differential Equations. Adv. Comp. Fluid Dynamics, Gordon Breach Publ. (1995) 307-331.

[16] J.J. Chattot, A Conservative Box-scheme for the Euler Equations. Int. J. Num. Meth. Fluids (to appear).

[17] J.J. Chattot and S. Malet, A "box-scheme" for the Euler equations. Lect. Notes Math. 1270, Springer-Verlag, Berlin (1987) $82-99$.

[18] Y. Coudière, J-P. Vila and P. Villedieu, Convergence rate of a finite volume scheme for a two dimensional convection-diffusion problem. Math. Model. Numer. 33 (1999) 493-516.

[19] B. Courbet, Schémas boîte en réseau triangulaire, Rapport technique 18/3446 EN (1992), ONERA, unpublished.

[20] B. Courbet, Schémas à deux points pour la simulation numérique des écoulements, La Recherche Aérospatiale $n^{\circ} 4$ (1990) $21-46$.

[21] B. Courbet, Étude d'une famille de schémas boîtes à deux points et application à la dynamique des gaz monodimensionnelle, La Recherche Aérospatiale $n^{\circ} 5$ (1991) 31-44.

[22] B. Courbet and J.P. Croisille, Finite Volume Box Schemes on triangular meshes. Math. Model. Numer. 32 (1998) 631-649.

[23] J-P. Croisille, Finite Volume Box Schemes, in Proc. of the 2nd Int. Symp. on Finite Volume for Complex Applications. Hermes, Paris (1999).

[24] M. Crouzeix and P.A. Raviart, Conforming and non conforming finite element methods for solving the stationary Stokes equations I. RAIRO 7 (1973) R-3, 33-76.

[25] F. Dubois, Finite volumes and mixed Petrov-Galerkin finite elements; the unidimensional problem. Num. Meth. PDE (to appear).

[26] R. Eymard, T. Gallouët and R. Herbin, Finite Volume Methods, in Handbook of Numerical Analysis, Ciarlet-Lions Eds. 5 (1997).

[27] G. Fairweather and R.D. Saylor, The reformulation and numerical solution of certain nonclassical initial-boundary value problems. SIAM J. Sci. Stat. Comput. 12 (1991) 127-144.

[28] L. Fezoui and B. Stoufflet, A class of implicit upwind schemes for Euler equations on unstructured grids. J. Comp. Phys. 84 (1989) 174-206.

[29] V. Girault and P.A. Raviart, Finite Element Approximation of the Navier-Stokes equations. Lect. Notes Math. 749, Springer, Berlin (1979).

[30] W. Hackbusch, On first and second order box schemes. Computing 41 (1989) 277-296.

[31] H.B. Keller, A new difference scheme for parabolic problems, Numerical solutions of partial differential equations, II, B. Hubbard Ed., Academic Press, New-York (1971) 327-350. 
[32] R.D. Lazarov, J.E. Pasciak and P.S. Vassilevski, Coupling mixed and finite volume discretizations of convection-diffusionreaction equations on non-matching grids, in Proc. of the 2nd Int. Symp. on Finite Volume for Complex Applications, Hermes, Paris (1999).

[33] L.D. Marini, An inexpensive method for the evaluation of the solution of the lowest order Raviart-Thomas mixed method. SIAM J. Numer. Anal. 22 (1985) 493-496.

[34] P.C. Meek and J. Norbury, Nonlinear moving boundary problems and a Keller box scheme. SIAM J. Numer. Anal. 21 (1984) 883-893.

[35] R.A. Nicolaides, Existence, uniqueness and approximation for generalized saddle point problems. SIAM J. Numer. Anal. 19 (1982) 349-357.

[36] P.A. Raviart and J.M. Thomas, A mixed finite element method for 2nd order elliptic problems. Lect. Notes Math. 606, Springer-Verlag, Berlin (1977) 292-315.

[37] E. Süli, Convergence of finite volume schemes for Poisson's equation on non-uniform meshes. SIAM J. Numer. Anal. 28 (1991) $1419-1430$.

[38] E. Süli, The accuracy of cell vertex finite volume methods on quadrilateral meshes. Math. of Comp. 59 (1992) 359-382.

[39] T. Schmidt, Box Schemes on quadrilateral meshes. Computing 51 (1993) 271-292.

[40] J-M Thomas and D. Trujillo, Mixed Finite Volume methods. Int. J. Num. Meth. Eng. 45 (1999) to appear.

[41] S.F. Wornom, Application of compact difference schemes to the conservative Euler equations for one-dimensional flows. NASA Tech. Mem. 83262 (1982).

[42] S.F. Wornom and M.M. Hafez, Implicit conservative schemes for the Euler equations. AIAA J. 24 (1986) 215-233.

[43] A. Younes, R. Mose, P. Ackerer and G. Chavent, A new formulation of the Mixed Finite Element Method for solving elliptic and parabolic PDE. J. Comp. Phys. 149 (1999) 148-167.

To access this journal online: www.edpsciences.org 